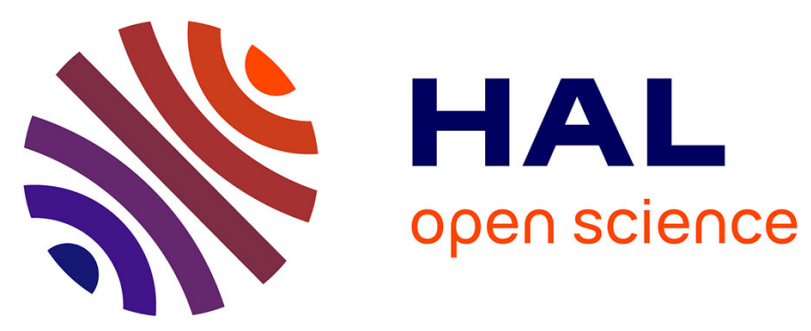

\title{
Targeting relaxase genes for classification of the predominant plasmids in Enterobacteriaceae
}

Fabrice Compain, Agathe Poisson, Simon Le Hello, Catherine Branger, François-Xavier Weill, Guillaume Arlet, Dominique Decré

\section{> To cite this version:}

Fabrice Compain, Agathe Poisson, Simon Le Hello, Catherine Branger, François-Xavier Weill, et al.. Targeting relaxase genes for classification of the predominant plasmids in Enterobacteriaceae. International Journal of Medical Microbiology Supplements, 2014, 304 (3-4), pp.236 - 242. 10.1016/j.ijmm.2013.09.009 . pasteur-01108896

\section{HAL Id: pasteur-01108896}

https://hal-pasteur.archives-ouvertes.fr/pasteur-01108896

Submitted on 12 Mar 2019

HAL is a multi-disciplinary open access archive for the deposit and dissemination of scientific research documents, whether they are published or not. The documents may come from teaching and research institutions in France or abroad, or from public or private research centers.
L'archive ouverte pluridisciplinaire HAL, est destinée au dépôt et à la diffusion de documents scientifiques de niveau recherche, publiés ou non, émanant des établissements d'enseignement et de recherche français ou étrangers, des laboratoires publics ou privés.

\section{(c)(1)}

Distributed under a Creative Commons Attribution| 4.0 International License 
1 Targeting relaxase genes for classification of the predominant plasmids in

2 Enterobacteriaceae

4 Fabrice Compain ${ }^{1}$, Agathe Poisson ${ }^{1}$, Simon Le Hello ${ }^{2}$, Catherine Branger $^{3}$, François5 Xavier Weill ${ }^{2}$, Guillaume Arlet ${ }^{1,4}$, Dominique Decré ${ }^{1,4} *$

$7 \quad{ }^{1}$ Université Pierre et Marie Curie-Paris 6, Faculté de Médecine, Site Saint-Antoine,

8 Laboratoire de Bactériologie, ER8, Paris, France; ${ }^{2}$ Institut Pasteur, Unité des Bactéries

9 Pathogènes Entériques, Centre National de Référence des Escherichia coli, Shigella et

Salmonella, WHO Collaborating Centre for Reference and Research on Salmonella, Paris,

France; ${ }^{3}$ Université Paris Diderot, UMR-S 722, Faculté de Médecine, Site Xavier Bichat, F-75018

Paris, France; ${ }^{4}$ Laboratoire de Bactériologie, Hôpital Saint-Antoine, Assistance Publique des Hôpitaux de Paris, Paris, France

*Corresponding author

Email addresses:

FC: compain.fabrice@wanadoo.fr

AP: agatepoisson@gmail.com

SLH: slehello@pasteur.fr

CB: catherine.branger@1mr.aphp.fr

FXW: fxweill@pasteur.fr

GA: guillaume.arlet@upmc.fr

DD: dominique.decre@ upmc.fr; Tel.: +33 14001 14 46; Fax +33 149282472 


\section{Abstract}

Plasmids are the main vectors of antimicrobial drug resistance and virulence genes, especially in Enterobacteriaceae. Identification and classification of plasmids is essential for analysis of their distribution. The most widely used typing method is PCR-based replicon typing (PBRT). A new classification scheme based on relaxase gene typing has been described recently. We propose a practical application of this method, with the development of a multiplex PCR set targeting relaxase genes found on plasmids most frequently encountered in

Enterobacteriaceae. This method, here called "plasmid relaxase gene typing" (PRaseT), was validated with 60 transconjugants and transformants harboring various replicon types. The method was tested with 39 multidrug-resistant clinical isolates including Escherichia coli, Klebsiella pneumoniae and Salmonella enterica subsp. enterica carrying 1 to 7 replicons as well as with 17 plasmids non-typeable using PBRT; all replicons were tested in parallel with PBRT for comparison.Six multiplex PCRs and one simplex PCR, including 24 pairs of primers, recognized plasmids of groups A/C, B/O, colE, FIA, FIB, FIC, FV, FIIk, HI1, HI2, I1, K, L/M, N, P1 $\alpha, \mathrm{Q} 1, \mathrm{U}, \mathrm{W}, \mathrm{X} 1, \mathrm{X} 2, \mathrm{X} 3$ and X4. There was perfect correlation between PRaseT and PBRT results in 31/39 (79.5\%) clinical isolates. Moreover, 11/17 (64.7\%) plasmids non-typeable by PBRT could be typed by PRaseT. Our set of multiplex PCRs showed high sensitivity and specificity for the classification of resistance plasmids. It has proved complementary to the widely used PBRT and will improve the monitoring of plasmid distribution in every-day practice.

\section{Keywords}

Plasmid; Classification; Replicon typing; Relaxase; Multiplex PCR 


\section{Introduction}

Plasmids are important agents of gene flux and have found to be responsible for the dissemination of multiple antibiotic resistance genes. Identification and classification of plasmids is essential for analysis of their distribution, their genetic relatedness and evolution, as well as for study of horizontal gene transfer. A classification scheme should be based on genetic traits that are universally present and constant. It should be robust and the corresponding experimental procedure should be easy. The basic replicon locus, which is always present on plasmids, has been used historically for classification. Plasmids were initially classified according to their incompatibility, which is directly related to replication. Incompatibility (Inc) was defined as the inability of two plasmids sharing common replication control (same Inc group) to be maintained in the subsequent lineage during conjugation (Datta and Hedges, 1971 and Novick, 1987). This method which requires plasmid transfer to the same host for testing is time-consuming and not practical for large-scale studies. Couturier et al. tested a method using hybridization with cloned replication regions as probes but with this method plasmid diversity is underestimated due to cross-hybridization (Couturier et al., 1988). In 2005, Carattoli et al. developed a PCR scheme of targeting replicons called PCRbased replicon typing (PBRT) (Carattoli et al., 2005). Eighteen pairs of primers were designed in order to perform 5 multiplex and 3 simplex PCRs recognizing the most frequently encountered plasmid incompatibility groups among Enterobacteriaceae. With this method, 27 Inc groups are currently recognized (Carattoli, 2009). It has been widely used to study plasmid spread and diversity in Enterobacteriaceae. However, PBRT has several drawbacks: (i) plasmids may carry multiple replicons and/or mosaic replicons, and new replicon types may escape classification with this technique; (ii) false-negative results with some Inc groups (e.g. L/M) have been reported (Carattoli et al., 2005) and (iii) PBRT targets multiple sites 
such as the replication initiation protein gene (rep), the active segregation partitioning system (par), replication control systems (iterons, antisense RNA), and recently also the relaxase gene (Carattoli et al., 2005 and Johnson et al., 2012), which may cause confusion. Plasmid multilocus sequence typing were also developed to refine classification of plasmid subgroups (García-Fernández and Carattoli, 2010, García-Fernández et al., 2008, García-Fernández et al., 2011 and Phan et al., 2009).

Bacterial conjugation represents a unique process allowing transfer of plasmid DNA from a donor to a recipient bacterium through cell-to-cell contact. In this process relaxase is a key protein encoded by all transmissible plasmids, i.e. mobilizable and conjugative plasmids involved in horizontal gene transfer (reviewed by Smillie et al., 2010 and Wong et al., 2012). A classification scheme based on the mobilization region of transmissible plasmids has recently been developed (Francia et al., 2004 and Garcillán-Barcia et al., 2009). The scheme classified relaxases in six protein families and 31 subfamilies, depending on their phylogeny; subsequently, 19 degenerate primer pairs targeting the relaxase genes of $\gamma$-proteobacterial plasmids were designed (Alvarado et al., 2012). This degenerate primer MOB typing (DPMT) method has been used with success in previous studies (Curiao et al., 2011, Mata et al., 2012, Mata et al., 2010 and Valverde et al., 2009). However, this set of primers was not designed for screening purposes in clinical practice, but rather for experimental purposes in order to discover new relaxases (Alvarado et al., 2012).

The aim of the present study was to design a multiplex PCR method, called "plasmid relaxase gene typing" (PRaseT), including novel oligonucleotide primers targeting relaxase genes of the plasmids most frequently encountered in Enterobacteriaceae in clinical practice. These multiplex PCRs were carried out under maximum consensus thermal cycling conditions, and applied to various plasmids present in clinical isolates from several collections. 


\section{Materials and methods}

\section{Database search and primer design}

An in silico analysis was carried out using GenBank BLAST (http://blast.ncbi.nlm.nih.gov/). For each Inc group, the relaxase/helicase gene was used as template; the presence of relaxasespecific multidomains was checked using CD-Search

(http://www.ncbi.nlm.nih.gov/Structure/cdd/wrpsb.cgi/). Multiple alignments were performed with ClustalW2 software (http://www.ebi.ac.uk/Tools/msa/clustalw2/). Primer pairs covering most sequences in each family were designed using FastPCR software (http://primerdigital.com/fastpcr.html), while minimizing codon degeneracy (Table 1A).

\section{In silico primer assay}

Oligonucleotide primers were tested in silico for hybridization with plasmids of the Enterobacteriaceae referenced in GenBank. Some primers were refined to cover a maximum of reported sequences.

\section{Bacterial strains}

For validation of the PCR assays, experiments were conducted with 60 Escherichia coli transconjugants or transformants of Enterobacteriaceae (Table 2). All strains carried replicons of various types that encoded diverse $\beta$-lactamases conferring resistance to third-generation cephalosporins or carbapenems. They were part of three collections of, respectively, (i) E. coli strains isolated between 1997 and 2002 in various French university hospitals (for further 
details see Marcadé et al., 2009 and Branger et al., 2005), (ii) Klebsiella pneumoniae strains from various geographical regions collected since the 1980s (D. Decré and G. Arlet, personal collection) and (iii) Salmonella enterica subsp. enterica strains representing various serovars (collection of the French National Reference Center for E. coli, Shigella, and Salmonella, Institut Pasteur). Assays with transconjugants and transformants for multiplex PCR optimization were carried out in triplicates.

All transconjugants, transformants and clinical strains used in this study were analyzed in parallel with PRaseT and the PBRT method applied previously (Carattoli et al., 2005, GarcíaFernández et al., 2009, Götz et al., 1996, Osborn et al., 2000 and Villa et al., 2010). The transconjugants used as positive controls in PRaseT reactions are given in Table 2. Other controls used in this study included three strains of the ECOR collection (Ochman and Selander, 1984) (ECOR 6, ECOR 10 and ECOR 19 harboring, respectively, pTPqnrS-1a-like, pcolE1-like- and IncX2-plasmids), as well as reference plasmids pFBAOT6 (IncU) (Rhodes et al., 2004) and RP4 (IncP1 $\alpha$ ) (Datta et al., 1971).

After optimization on transconjugants or transformants carrying replicon of various types according to PBRT, we applied the PRaseT method to a panel of 39 clinical strains (21E. coli, $16 \mathrm{~K}$. pneumoniae and 2 S. enterica) carrying replicons of one to seven different types (Table 3), and 17 transconjugants or transformants that were non-typeable with PBRT (Table 4).

\section{DNA extraction and PCR conditions}

InstaGene matrix (Biorad, Marnes la Coquette, France) or lysis by boiling were used for total DNA extraction as previously described (Dallenne et al., 2010). Multiplex PCR was carried out using the Qiagen Multiplex PCR Kit (Qiagen, Courtaboeuf, France). The master mix 
contained pre-optimized concentrations of HotStarTaq DNA polymerase and $\mathrm{MgCl}_{2}$, deoxynucleotide triphosphate and PCR buffer. To all multiplex PCRs, solution Q (Qiagen) that facilitates the reaction with difficult-to-amplify templates by modifying DNA melting behavior was added. Total DNA in $5 \mu$ l of bacterial lysate was subjected to multiplex PCR in a $50 \mu 1$ volume. The conditions for multiplex PCR were optimized to ensure that all targets were sufficiently amplified for amplicons to be easily visible on $1.5 \%$ agarose gels. The optimal primer concentrations are reported in Table 1A. PCR conditions consisted in initial activation at $95^{\circ} \mathrm{C}$ for $15 \mathrm{~min}$, followed by 30 cycles at $94^{\circ} \mathrm{C}$ for $30 \mathrm{~s}, 60^{\circ} \mathrm{C}$ for $90 \mathrm{~s}$ and $72^{\circ} \mathrm{C}$ for $90 \mathrm{~s}$ with a final extension at $72^{\circ} \mathrm{C}$ for $10 \mathrm{~min}$; for Multiplex IV and $\mathrm{V}$ the annealing temperature was elevated to $65^{\circ} \mathrm{C}$ for $90 \mathrm{sec}$. Simplex PCR were performed in a $50 \mu \mathrm{L}$ mix with $2 \mathrm{U}$ of Taq DNA polymerase (Roche Diagnostics), $10 \times \mathrm{PCR}$ buffer/ $\mathrm{MgCl}_{2}$ (Roche Diagnostics), $200 \mu \mathrm{M}$ of each deoxynucleotide triphosphate (dNTP Mix Eurobio), 0.2 $\mathrm{pmol} / \mu \mathrm{L}$ of each primer, $40 \mu \mathrm{L}$ of sterile water and $2 \mu \mathrm{L}$ of total DNA extract. PCR conditions consisted in 30 cycles $\left[94^{\circ} \mathrm{C}\right.$ for $1 \mathrm{~min}, 55^{\circ} \mathrm{C}$ for $40 \mathrm{sec}, 72^{\circ} \mathrm{C}$ for $1 \mathrm{~min}$ ], preceded by one cycle at $94^{\circ} \mathrm{C}$ for $5 \mathrm{~min}$ followed by one cycle at $72^{\circ} \mathrm{C}$ for $5 \mathrm{~min}$. PCR products were separated at $100 \mathrm{~V}$ for 90 minutes in $1.5 \%$ agarose gel containing ethidium bromide and visualized using GelDoc (Biorad). PCR products were purified using the Exosap purification kit (illustra ExoStar 1-Step, Dutscher, Brumath, France) and subjected to bidirectional DNA sequencing using the BigDye terminator v3.1 cycle sequencing kit (Applied Biosystems, Foster City, CA, USA) and an Applied Biosystems 3730 XL capillary sequencer. Sequence analysis was carried out using BLAST and GenBank sequences.

\section{Results and discussion}

\section{In silico analysis}


We mainly focused on plasmid families previously found to be involved in the spread of resistance genes in Enterobacteriaceae (Carattoli, 2013, 2011 and 2009). Since no complete sequence of IncFIII, IncFIV, IncFVI, IncFVII and IncY plasmids have been reported and their sequenced segments do not contain relaxase genes, these plasmids were excluded from this study. So were those of the IncR family which is known not to contain any relaxase gene (Alvarado et al., 2012) and those of the IncJ family and R391-like elements that are part of integrative and conjugative elements (Burrus et al., 2006) the analysis of which is beyond the scope of this study. Also not considered here were some relatively rare groups (e.g. IncI2 plasmids) or poorly resolved groups (e.g. IncQ3 or IncT plasmids) (Alvarado et al., 2012).

The majority of plasmids had a single putative relaxase locus, which is consistent with the review by Smillie et al. (Smillie et al., 2010). In contrast, complete GenBank sequences of IncHI1 and IncHI2 plasmids had one to three putative relaxase/helicase loci on each reported plasmid (Supplementary Table S1). CD-Search results for each protein confirmed the presence of relaxase- or helicase-specific multidomains. We finally used five relaxase clades (arbitrarily designed HI $\alpha, \mathrm{HI} \beta, \mathrm{HI} \gamma, \mathrm{HI} \delta$ and $\mathrm{HI} \varepsilon$ ) present among the various IncHI1 and/or IncHI2 plasmids (Supplementary Table S1). HI $\beta$ and HI $\delta$ relaxases were found to be encoded only on IncHI1 plasmids and HI $\gamma$ and HIe relaxases only on IncHI2 plasmids while HIa relaxases were encoded on both IncHI1 and IncHI2 plasmids. We therefore designed three oligonucleotide primers covering the $\mathrm{HI} \alpha, \mathrm{HI} \beta$ and $\mathrm{HI} \gamma$ relaxase, respectively.

In silico analysis led to the design of 24 pairs of primers for six multiplex PCRs (targeting the relaxase genes of plasmids belonging to Inc groups A/C, colE, FIA, FIB, FIC, FII, FIIk, FV, HI1, HI2, I1, L/M, N, P1 $\alpha, \mathrm{Q} 1, \mathrm{U}, \mathrm{W}, \mathrm{X} 1, \mathrm{X} 2, \mathrm{X} 3$ and X4) and one simplex PCR (targeting the relaxase genes of plasmids belonging to Inc groups B/O and K) (Table 1A). 


\section{Primer evaluation using transconjugants and transformants}

In order to assess the sensitivity and specificity of each PCR, primers were tested using a collection of 60 recipient cells, with PBRT as the reference method (Table 2). Each primer pair was validated using all recipient cells, first in a simplex and then a multiplex PCR and target DNA of either single cells or cell mixtures was used. PCR conditions were optimized and all amplicons were sequenced. E. coli strain J53 was used as negative control in PCR experiments to test for possible cross-hybridization with chromosomal DNA. No non-specific amplification was observed.

All PRaseT results were consistent with the PBRT results, except for two strains which carried an IncR (K. pneumoniae strain S51) or an IncFIB/FII replicon (E. coli strain 81), that were undetected by the PRaseT method. For the first strain the result was not unexpected as IncR plasmids do not encode relaxases. For the second strain, the result was more surprising as IncF plasmids are known to be conjugative in most cases (Smillie et al., 2010). As it has been previously reported that Mob regions and mating pair formation (MPF) systems were in general of the same type (Smillie et al., 2010), we designed new primers targeting other conserved genes of the type IV secretion system (T4SS), i.e. traB (encoding a secretin-like protein) and traC (encoding an ATPase) (Supplementary Table S2). The eighteen PRaseTpositive IncF plasmids reported in Table 2 tested positive for $\operatorname{tr} a B$ and $\operatorname{tra} C$ while the PRaseTnegative IncFIB/FII plasmid also tested negative with T4SS typing (data not shown). Interestingly, no transconjugant but only transformant was obtained from the parental strain of the later plasmid. We considered three possibilities: (i) a very divergent IncF relaxase gene that could not hybridize with our primers was present, (ii) the relaxase gene was truncated or (iii) the gene was absent. Complete sequencing of the plasmid will be performed to confirm one of these possibilities. 
For IncHI plasmids, three primer sets were mandatory to differentiate IncHI1 from IncHI2. As noted above, in most cases, the identification of relaxases from IncHI1 and IncHI2 plasmids was obtained with positive results for HI $\beta$ - and $\mathrm{HI} \gamma$-primers respectively (e.g. transconjugants S01477 and 102, Table 2). However, when Hia PCR was the only positive result (e.g. IncHI2containing Salmonella S09118), we used T4SS-typing as a complement to PRaseT (Supplementary Table S2). The positive result of PCR targeting the T4SS from IncHI2 plasmids confirmed the presence of a HI2 relaxase in S09118.

\section{Evaluation of relaxase gene typing using clinical strains}

To further confirm the specificity of the designed primer set, 39 clinical strains, each carrying from one to seven different replicon types, were submitted to PRaseT (Table 3). An example of the results is shown in Fig. 1. For 31 strains $(79.5 \%)$ there was a perfect correlation between the results obtained with PRaseT and PBRT.

Five strains were positive with PBRT but negative with PRaseT. Among these, two (E. coli strains 19 and 34, Table 3) carried IncFIA/FIB/FII replicons. PCR targeting the T4SS genes $\operatorname{tra} B$ and $\operatorname{traC}$ was negative, and the plasmid from neither strain could be transferred to a recipient cell by conjugation. Similarly, for the IncU plasmid of strain KpS15 tested negative with PRaseT, primers targeting virB4, the gene coding for the ATPase of the T4SS of IncU plasmids (Supplementary Table 2), were used. The result was negative. The two remaining strains (i.e E. coli 33 and 101) carried an IncP plasmid which could not be typed by PRaseT. Our primer pair was designed using the reference plasmid RP4 as template. However, this plasmid belongs to the IncP1 $\alpha$ subgroup, while the IncP1 group consists of at least six 
divergent subgroups (IncP1 $\alpha, \operatorname{IncP} 1 \beta$, IncP $1 \gamma, \operatorname{IncP} 1 \delta$, IncP1 $\varepsilon$ and IncP1 $\zeta$ ), many of which are antibiotic resistance vectors in the environment (Alvarado et al., 2012, Bahl et al., 2009 and Heuer et al., 2012). We think that our IncP1 $\alpha$ relaxase primers were unable to classify all IncP1 group replicons because of too great a divergence in gene sequences. PRaseT of IncP group plasmids should be improved in further studies.

Finally, three strains (K. pneumoniae KpS63 and FM10, and E. coli 105) which were negative with PBRT (after multiple PCR assays) were found to contain IncFIIk, IncHI and IncI1 plasmids, respectively, when PRaseT and T4SS typing was used (Table 3). The presence of sequence divergence or mosaic replicons may explain these results that will be clarified by sequencing. These observations underscore the complementarity between the PBRT and PRaseT methods.

\section{Relaxase gene typing in recipient cells non-typeable with PBRT}

Seventeen recipient cells whose plasmids were found to be non-typeable with PBRT were subjected to typing with PRaseT; parental strains included 14 strains of E. coli and 3 of $K$. pneumoniae. PCR results are given in Table 4. In the 17 recipient cells, 11 (64.7\%) plasmids, mainly pHUSEC41-4-like mobilizable replicons, could be typed with PRaseT. The complete sequence determination of plasmids from 6 recipient cells (i.e. E. coli strains 65, 66, 70, 71, 72 and 99) confirmed these results (unpublished data). The majority of bla $a_{\mathrm{SHV}}$ ESBL genes (six out of eight) were localized on pHUSEC41-4-like plasmids. Such colE-like plasmids have been already reported as resistance vectors in Enterobacteriaceae (García-Fernández et al., 2009). 
Six plasmids could be typed neither with PBRT nor with PRaseT (Table 4). Complete sequence is available for 3 of them, i.e. plasmids from K. pneumoniae transformant S77 (pKpS77, SHV-12), E. coli transconjugant 76 (RCS47v1_pI, SHV-2) and E. coli transformant 93 (RCS63v1_p, CTX-M-3) (unpublished data). Plasmids pKpS77, RCS47v1_pI and RCS63v1_p were, respectively, $<50,000$ bp, 117,001 bp and 22,308 bp long. In the sequences of two of them (pKpS77 and RCS63v1_p), no transfer region could be identified. The third plasmid (RCS47v1_pI, E. coli transconjugant 76) carried a new relaxase gene which presented $100 \%$ query cover and $99 \%$ maximum identity with those on plasmids pO111_2 (GenBank accession no. AP010962.1) and p12579_1 (GenBank accession no. CP003110.1); its replicase gene also presented $100 \%$ query cover and $99 \%$ maximum identity with its counterparts on plasmids pO111_2 and p12579_1. The three other non-typeable plasmids (contained in transformants from K. pneumoniae S33, E. coli 55 and E. coli 86) could not be transferred by conjugation, which is in favor of non-transmissible plasmids; this should, however, be confirmed by complete plasmid sequencing.

\section{Conclusions}

Our set of six multiplex PCRs and one simplex PCR allowed classification of the most frequently encountered transmissible plasmids in Enterobacteriaceae by targeting their relaxase gene. It stands as a promising complement to the widely used PBRT method in understanding plasmid spread and evolution, and can be applied to epidemiological surveys as well. Considering the complexity of constant plasmid evolution, the combined use of two complementary and practical classification tools should be advantageous and reduce the need for systematic full-length plasmid sequencing. 
282 Competing interest

283 The authors declare that they have no competing interests.

284

285 Acknowledgements

286 We are grateful to Etienne Carbonnelle (Hôpital Européen Georges Pompidou, Paris, France)

287 for providing the three control strains from the ECOR collection, and to Glenn Rhodes

288 (Lancaster Environment Centre, Lancaster, United Kingdom) for providing the IncU-positive 289 control strain. 


\section{References}

Alvarado A, Garcillán-Barcia MP, de la Cruz F, 2012. A degenerate primer MOB typing (DPMT) method to classify gamma-proteobacterial plasmids in clinical and environmental settings. PLoS One. 7 (7), e40438.

Bahl MI, Burmølle M, Meisner A, Hansen LH, Sørensen SJ, 2009. All IncP-1 plasmid subgroups, including the novel epsilon subgroup, are prevalent in the influent of a Danish wastewater treatment plant. Plasmid. 62 (2), 134-139.

Branger C, Zamfir O, Geoffroy S, Laurans G, Arlet G, Thien HV, Gouriou S, Picard B, Denamur E, 2005. Genetic background of Escherichia coli and extended-spectrum beta-lactamase type. Emerg Infect Dis. 11 (1), 54-61.

Burrus V, Marrero J, Waldor MK, 2006. The current ICE age: biology and evolution of SXT-related integrating conjugative elements. Plasmid. 55 (3), 173-183.

Carattoli A, 2009. Resistance plasmid families in Enterobacteriaceae. Antimicrob Agents Chemother. $53(6), 2227-2238$.

Carattoli A, 2011. Plasmids in Gram negatives: molecular typing of resistance plasmids. Int J Med Microbiol. 301 (8), 654-658.

Carattoli A, 2013. Plasmids and the spread of resistance. Int J Med Microbiol. S1438-4221(13).

Carattoli A, Bertini A, Villa L, Falbo V, Hopkins KL, Threlfall EJ, 2005. Identification of plasmids by PCRbased replicon typing. J Microbiol Methods. 63 (3), 219-228.

Couturier M, Bex F, Bergquist PL, Maas WK, 1988. Identification and classification of bacterial plasmids. Microbiol Rev. 52 (3), 375-395.

Curiao T, Cantón R, Garcillán-Barcia MP, de la Cruz F, Baquero F, Coque TM, 2011. Association of composite IS26-sul3 elements with highly transmissible Incl1 plasmids in extended-spectrumbeta-lactamase-producing Escherichia coli clones from humans. Antimicrob Agents Chemother. 55 (5), 2451-2457.

Dallenne C, Da Costa A, Decré D, Favier C, Arlet G., 2010. Development of a set of multiplex PCR assays for the detection of genes encoding important beta-lactamases in Enterobacteriaceae. J Antimicrob Chemother. 65 (3), 490-495.

Datta N, Hedges RW, 1971. Compatibility groups among fi - R factors. Nature. 234 (5326), 222-223.

Datta N, Hedges RW, Shaw EJ, Sykes RB, Richmond MH, 1971. Properties of an R factor from Pseudomonas aeruginosa. J Bacteriol. 108 (3), 1244-1249.

Francia MV, Varsaki A, Garcillán-Barcia MP, Latorre A, Drainas C, de la Cruz F, 2004. A classification scheme for mobilization regions of bacterial plasmids. FEMS Microbiol Rev. 28 (1), 79-100.

García-Fernández A, Carattoli A, 2010. Plasmid double locus sequence typing for IncHI2 plasmids, a subtyping scheme for the characterization of IncHI2 plasmids carrying extended-spectrum beta-lactamase and quinolone resistance genes. J Antimicrob Chemother. 65 (6), 1155-1161.

García-Fernández A, Chiaretto G, Bertini A, Villa L, Fortini D, Ricci A, Carattoli A, 2008. Multilocus sequence typing of Incl1 plasmids carrying extended-spectrum beta-lactamases in Escherichia coli and Salmonella of human and animal origin. J Antimicrob Chemother. 61 (6), 1229-1233.

García-Fernández A, Fortini D, Veldman K, Mevius D, Carattoli A, 2009. Characterization of plasmids harbouring qnrS1, qnrB2 and qnrB19 genes in Salmonella. J Antimicrob Chemother. 63 (2), 274-281.

García-Fernández A, Villa L, Moodley A, Hasman H, Miriagou V, Guardabassi L, Carattoli A, 2011. Multilocus sequence typing of IncN plasmids. J Antimicrob Chemother. 66 (9), 1987-1991.

Garcillán-Barcia MP, Francia MV, de la Cruz F, 2009. The diversity of conjugative relaxases and its application in plasmid classification. FEMS Microbiol Rev. 33 (3), 657-687. 
Götz A, Pukall R, Smit E, Tietze E, Prager R, Tschäpe H, van Elsas JD, Smalla K, 1996. Detection and characterization of broad-host-range plasmids in environmental bacteria by PCR. Appl Environ Microbiol. 62 (7), 2621-2628.

Heuer H, Binh CT, Jechalke S, Kopmann C, Zimmerling U, Krögerrecklenfort E, Ledger T, González B, Top E, Smalla K, 2012. IncP-1ع Plasmids are Important Vectors of Antibiotic Resistance Genes in Agricultural Systems: Diversification Driven by Class 1 Integron Gene Cassettes. Front Microbiol. 3 (2).

Johnson TJ, Bielak EM, Fortini D, Hansen LH, Hasman H, Debroy C, Nolan LK, Carattoli A, 2012. Expansion of the IncX plasmid family for improved identification and typing of novel plasmids in drug-resistant Enterobacteriaceae. Plasmid. 68 (1), 43-50.

Marcadé G, Deschamps C, Boyd A, Gautier V, Picard B, Branger C, Denamur E, Arlet G, 2009. Replicon typing of plasmids in Escherichia coli producing extended-spectrum beta-lactamases. J Antimicrob Chemother. 63 (1), 67-71.

Mata C, Miró E, Alvarado A, Garcillán-Barcia MP, Toleman M, Walsh TR, de la Cruz F, Navarro F, 2012. Plasmid typing and genetic context of AmpC $\beta$-lactamases in Enterobacteriaceae lacking inducible chromosomal ampC genes: findings from a Spanish hospital 1999-2007. J Antimicrob Chemother. 67 (1), 115-122.

Mata C, Miró E, Mirelis B, Garcillán-Barcia MP, de la Cruz F, Coll P, Navarro F, 2010. In vivo transmission of a plasmid coharbouring bla and qnrB genes between Escherichia coli and Serratia marcescens. FEMS Microbiol Lett. 308 (1), 24-28.

Novick RP, 1987. Plasmid incompatibility. Microbiol Rev. 51 (4), 381-395.

Ochman H, Selander RK, 1984. Standard reference strains of Escherichia coli from natural populations. J Bacteriol. 157 (2), 690-693.

Osborn AM, da Silva Tatley FM, Steyn LM, Pickup RW, Saunders JR, 2000. Mosaic plasmids and mosaic replicons: evolutionary lessons from the analysis of genetic diversity in IncFII-related replicons. Microbiology. 146 (9), 2267-2275.

Phan MD, Kidgell C, Nair S, Holt KE, Turner AK, Hinds J, Butcher P, Cooke FJ, Thomson NR, Titball R, Bhutta ZA, Hasan R, Dougan G, Wain J, 2009. Variation in Salmonella enterica serovar typhi IncHI1 plasmids during the global spread of resistant typhoid fever. Antimicrob Agents Chemother. 53 (2), 716-727.

Rhodes G, Parkhill J, Bird C, Ambrose K, Jones MC, Huys G, Swings J, Pickup RW, 2004. Complete nucleotide sequence of the conjugative tetracycline resistance plasmid pFBAOT6, a member of a group of IncU plasmids with global ubiquity. Appl Environ Microbiol. 70 (12), 7497-7510.

Smillie C, Garcillán-Barcia MP, Francia MV, Rocha EP, de la Cruz F, 2010. Mobility of plasmids. Microbiol Mol Biol Rev. 74 (3), 434-452.

Valverde A, Cantón R, Garcillán-Barcia MP, Novais A, Galán JC, Alvarado A, de la Cruz F, Baquero F, Coque TM, 2009. Spread of bla(CTX-M-14) is driven mainly by IncK plasmids disseminated among Escherichia coli phylogroups A, B1, and D in Spain. Antimicrob Agents Chemother. 53 (12), 5204-5212.

Villa L, García-Fernández A, Fortini D, Carattoli A, 2010. Replicon sequence typing of IncF plasmids carrying virulence and resistance determinants. J Antimicrob Chemother. 65 (12), 25182529.

Wong JJ, Lu J, Glover JN, 2012. Relaxosome function and conjugation regulation in F-like plasmids - a structural biology perspective. Mol Microbiol. 85 (4), 602-617. 
Tables

Table 1 - Primers used in this study

\begin{tabular}{|c|c|c|c|c|c|c|c|c|}
\hline PCR name & Plasmid type detected & Primer name & Sequence $\left(5^{\prime}-3^{\prime}\right)$ & Plasmid prototype & EMBL accession No. & Target site & Amplicon size (bp) & Primer concentration $(\mathrm{pmol} / \mu \mathrm{L})$ \\
\hline \multirow{6}{*}{ Multiplex I } & IncFIIK & MRxeFII-K_for & CGATATTCTKGAACCCCGTA & pKPN3 & CP000648.1 & tral & 297 & 0.2 \\
\hline & & MRxeFII-K_rev & TCATTGCCCATAATYCGTCC & & & & & 0.2 \\
\hline & IncFIA, IncFIB, IncFII & MRxeF-tot_for & ATCAGGAMCCACAGTTACAC & R100 & AP000342.1 & tral & 753 & 0.2 \\
\hline & & MRxeF-tot_rev & GTTTCATGATRTCRCGACTGAG & & & & & 0.2 \\
\hline & IncFV & MRxeFV_for & CATCAAAGCGAARGAGYAGTTCAC & pED208 & AF411480.1 & tral & 642 & 0.2 \\
\hline & & MRxeFV_rev & TTACCCTCWGCAATATGRCGRA & & & & & 0.2 \\
\hline \multirow{8}{*}{ Multiplex II } & $\operatorname{IncN}$ & MRxeN_for & CCAGTTTAGAAACCCGATCA & R46 & AY046276.1 & tral & 332 & 0.2 \\
\hline & & MRxeN_rev & CGTAATCGTCGTAAATGCTG & & & & & 0.2 \\
\hline & Incl1 & MRxel1_for & TTTAATCATGAGCAAACGCC & R64 & АВ027308.1 & nikB & 764 & 0.2 \\
\hline & & MRxel1_rev & ACAACYCTGTCACCATAATC & & & & & 0.2 \\
\hline & IncL/M & MRxeLM_for & TCGCTATATCGTTGGTGATG & pCTX-M3 & AF550415.2 & nikB & 491 & 0.2 \\
\hline & & MRxeLM_rev & GGGTATGTTGGGCAATTTTC & & & & & 0.2 \\
\hline & $\operatorname{lncA} / \mathrm{C}$ & MRxeAC_for & TGATTGAGAAAGTGCGGAACTC & pRA1 & FJ705807.1 & tral & 659 & 0.2 \\
\hline & & MRxeAC_rev & CACACCATAGGAGAACTCGT & & & & & 0.2 \\
\hline \multirow{6}{*}{ Multiplex III } & IncHI1, IncHI2 & MRxeHIa_for & CCAAAAGACTGACTTGGGAGCA & pMAK1 & AB366440.1 & helicase & 873 & 0.2 \\
\hline & & MRxeHIa_rev & CCGAGTTAGCCATCAAGGAA & & & & & 0.2 \\
\hline & IncHI1 & MRxeHIß_for & GATGGTATGTGGATAGTCCG & pNDM-CIT & JX182975.1 & tral & 368 & 0.2 \\
\hline & & MRxeHIB_rev & GAATATGCCTCACYATCGCT & & & & & 0.2 \\
\hline & IncHI2 & MRxeHly for & AATGACGTGGTTGAATACGA & R478 & BX664015.1 & tral & 225 & 0.2 \\
\hline & & MRxeHIY_rev & AGAATAGAAATCAGCGGTCC & & & & & 0.2 \\
\hline \multirow{8}{*}{ Multiplex IV } & IncX1 & MRxeX1_for & GAAAATGGTCGTAAGTCAGCT & pOLA52 & EU370913.1 & $\operatorname{tax} \mathrm{C}$ & 845 & 0.2 \\
\hline & & MRxeX1_rev & TTCAGYCTGACAAGATCACCTGC & & & & & 0.2 \\
\hline & $\operatorname{lnc} \times 2$ & MRxeX2_for & CGAAAATGGTCGTAAGTCTGCA & R6K & X95535.1 & $\operatorname{tax} C$ & 560 & 0.2 \\
\hline & & MRxeX2_rev & CATTACAAAAGCCCGTCCTG & & & & & 0.2 \\
\hline & $\operatorname{lnc} \times 3$ & MRxeX3_for & TGTTGACAAGGAATTCAGGGT & plncX-SHV & $\mathrm{JN} 247852.1$ & $\operatorname{tax} C$ & 736 & 0.2 \\
\hline & & MRxeX3_rev & ATGGTCGTAGCCAACATCAA & & & & & 0.2 \\
\hline & $\operatorname{IncX} 4$ & MRxeX4_for & TTAAACGCGCTAAAGGGACTGG & pSH146_32 & $J \times 258655.1$ & $\operatorname{tax} C$ & 996 & 0.2 \\
\hline & & MRxeX4_rev & GGGCAATATTTTCAATCTGCCAG & & & & & 0.2 \\
\hline \multirow{8}{*}{ Multiplex V } & Non typeable & MRxeMobC11 for & ACGGATTCGCTTRCCTGGMAGTG & pColEST258 & JN247853.1 & mobB & 997 & 0.2 \\
\hline & & MRxeMobC11_rev & TTCAGSGCGTACTGGTCGAGATCG & & & & & 0.2 \\
\hline & Non typeable & MRxeMobQu for & TCTCCAGAARCAGCGGCTACAG & pIGWZ12 & DQ311641.1 & mob & 481 & 0.04 \\
\hline & & MRxeMobQu_rev & ATGGTCAGCCCATGATGCACGCCA & & & & & 0.04 \\
\hline & colE, Non typeable & MRxeMobP5-1 for & AGTCCGGGARCTYATYGATGCCAC & pCOLE1 & J01566.1 & mob3 & 367 & 0.04 \\
\hline & & MRxeMobP5-1_rev & TATCAGRGAGWGTCAGYRTCCKGC & & & & & 0.04 \\
\hline & colE, Non typeable & MRxeMobP5-2_for & TCCGGTGGAYTATCTKCTGGGKA & pTPqnrS-1a & AM746977.1 & mobA & 656 & 0.2 \\
\hline & & & & -16 & & & & \\
\hline
\end{tabular}




\begin{tabular}{|c|c|c|c|c|c|c|c|c|}
\hline & colE, Non typeable & $\begin{array}{l}\text { MRxeMobP5-2_rev } \\
\text { MRxeMobP5-3_for } \\
\text { MRxeMobP5-3_rev }\end{array}$ & $\begin{array}{l}\text { AAGACTGYTCATARATRGCTCC } \\
\text { GCTATCAGTCGCCCTGTTCGTC } \\
\text { AGTACCCGATCATCAGTCAGTG }\end{array}$ & pHUSEC41-4 & HE603113.1 & $\mathrm{mob}$ & 147 & $\begin{array}{l}0.2 \\
0.04 \\
0.04\end{array}$ \\
\hline \multirow{8}{*}{ Multiplex VI } & IncQ1 & MRxeQ1_for & GATTTATCACCTTACGGCGAA & RSF1010 & M28829.1 & mobA & 962 & 0.2 \\
\hline & & MRxeQ1_rev & TACCTCCCGCAACTCTTTGG & & & & & 0.2 \\
\hline & $\operatorname{IncU}$ & MRxeU_for & TATGACACTCTAGCCGGGTT & pRA3 & DQ401103.1 & nic & 743 & 0.2 \\
\hline & & MRxeU_rev & TGGATCTTGTCTTCCCACGGTT & & & & & 0.2 \\
\hline & $\operatorname{IncP} 1 \alpha$ & MRxeP1a_for & TGAAGTACATCACCGACGAG & RP4 & X54459.1 & tral & 424 & 0.2 \\
\hline & & MRxeP1a_rev & GTTTCGTGATTGTCACGCTC & & & & & 0.2 \\
\hline & IncW & MRxeW_for & CATCGAGTTCGATACCCTTG & R388 & BR000038.1 & trwc & 531 & 0.2 \\
\hline & & MRxeW_rev & GCCACATAGTAAACGTCCTT & & & & & 0.2 \\
\hline \multirow{2}{*}{ Simplex I } & IncB/O, IncK & MRxeBO/K_for & GAATGCCATYATTCCGMAMAA & p0113 & AY258503.2 & nikB & 800 & 0.2 \\
\hline & & MRxeBO/K_rev & GTGATATACAGACCATCACTGG & & & & & 0.2 \\
\hline
\end{tabular}


Table 2 - Recipient cells used in validation experiments ${ }^{\star}$

\begin{tabular}{|c|c|c|c|c|c|}
\hline \multirow[b]{2}{*}{ No. } & \multirow[b]{2}{*}{ Parental strain } & \multirow[b]{2}{*}{$\mathrm{Tc} / \mathrm{Tf}$} & \multirow[b]{2}{*}{$\beta$-Lactamase } & \multicolumn{2}{|c|}{ Plasmid classification } \\
\hline & & & & PBRT & PRaseT \\
\hline $6 \dagger$ & E. coli & Tc & TEM-10 & $\mathrm{L} / \mathrm{M}$ & $\mathrm{L} / \mathrm{M}$ \\
\hline $40 \dagger$ & E. coli & $\mathrm{Tc}$ & TEM-24 & $\mathrm{A} / \mathrm{C}$ & $\mathrm{A} / \mathrm{C}$ \\
\hline $44 \dagger$ & E. coli & $\mathrm{Tc}$ & TEM-52 & I1 & I1 \\
\hline $48 \dagger$ & E. coli & $\mathrm{Tc}$ & TEM-52 & $\mathrm{X} 1$ & $\mathrm{X} 1$ \\
\hline 50 & E. coli & $\mathrm{Tc}$ & TEM-52 & I1 & I1 \\
\hline 52 & E. coli & $\mathrm{Tf}$ & TEM-52 & $\mathrm{X} 1$ & $\mathrm{X} 1$ \\
\hline 51 & E. coli & $\mathrm{Tc}$ & TEM-3 & $\mathrm{L} / \mathrm{M}$ & $\mathrm{L} / \mathrm{M}$ \\
\hline $57 \dagger$ & E. coli & $\mathrm{Tc}$ & SHV-12 & $\mathrm{F}$ & $\mathrm{F}$ \\
\hline 62 & E. coli & $\mathrm{Tc}$ & SHV-2 & FIB & $\mathrm{F}$ \\
\hline $64 \dagger$ & E. coli & $\mathrm{Tc}$ & SHV-5 & $\mathrm{X} 4$ & $\mathrm{X} 4$ \\
\hline 73 & E. coli & $\mathrm{Tc}$ & SHV-4 & FIIK & FIIK \\
\hline 81 & E. coli & Tf & CTX-M-3 & FIB, FII & - \\
\hline 85 & E. coli & $\mathrm{Tf}$ & CTX-M-1 & FIA & $\mathrm{F}$ \\
\hline $91 \dagger$ & E. coli & $\mathrm{Tc}$ & CTX-M-3 & $\mathrm{N}$ & $\mathrm{N}$ \\
\hline 98 & E. coli & Tc & CTX-M-1 & $\mathrm{X} 1$ & $\mathrm{X} 1$ \\
\hline 100 & E. coli & $\mathrm{Tc}$ & CTX-M-1 & FIA, FII & $\mathrm{F}$ \\
\hline $102 \dagger$ & E. coli & $\mathrm{Tc}$ & CTX-M-1 & HI2 & $\mathrm{HI} \alpha, \mathrm{HI} \gamma$ \\
\hline 104 & E. coli & Tf & CTX-M-1 & FIA, FIB, FII & $\mathrm{F}$ \\
\hline 105 & E. coli & $\mathrm{Tc}$ & CTX-M-1 & $\mathrm{L} / \mathrm{M}$ & $\mathrm{L} / \mathrm{M}$ \\
\hline 108 & E. coli & $\mathrm{Tf}$ & CTX-M-1 & FIA & $\mathrm{F}$ \\
\hline 110 & E. coli & $\mathrm{Tc}$ & CTX-M-1 & $\mathrm{X} 4$ & $\mathrm{X} 4$ \\
\hline 111 & E. coli & $\mathrm{Tc}$ & CTX-M-1 & FIA & $\mathrm{F}$ \\
\hline 114 & E. coli & $\mathrm{Tc}$ & CTX-M-1 & $\mathrm{L} / \mathrm{M}$ & $\mathrm{L} / \mathrm{M}$ \\
\hline 118 & E. coli & $\mathrm{Tc}$ & CTX-M-1 & FIA, FIB & $\mathrm{F}$ \\
\hline 120 & E. coli & $\mathrm{Tc}$ & CTX-M-3 & FIB & $\mathrm{F}$ \\
\hline 125 & E. coli & $\mathrm{Tc}$ & CTX-M-1 & FII & $\mathrm{F}$ \\
\hline 126 & E. coli & $\mathrm{Tc}$ & CTX-M-1 & $\mathrm{N}$ & $\mathrm{N}$ \\
\hline 127 & E. coli & $\mathrm{Tc}$ & CTX-M-3 & FIB & $\mathrm{F}$ \\
\hline S6 & K. pneumoniae & $\mathrm{Tc}$ & SHV-5 & $\mathrm{A} / \mathrm{C}$ & $\mathrm{A} / \mathrm{C}$ \\
\hline $\mathrm{S} 9+$ & K. pneumoniae & $\mathrm{Tc}$ & SHV-4 & FIIK & FIIK \\
\hline S16 & K. pneumoniae & $\mathrm{Tc}$ & CTX-M-3 & $\mathrm{A} / \mathrm{C}$ & $\mathrm{A} / \mathrm{C}$ \\
\hline S19 & K. pneumoniae & $\mathrm{Tc}$ & CTX-M-15/OXA-1 & $\mathrm{N}$ & $\mathrm{N}$ \\
\hline $\mathrm{S} 23$ & K. pneumoniae & $\mathrm{Tc}$ & CTX-M-3 & $\mathrm{N}, \mathrm{A} / \mathrm{C}$ & $\mathrm{N}, \mathrm{A} / \mathrm{C}$ \\
\hline $\mathrm{S} 24$ & K. pneumoniae & $\mathrm{Tc}$ & TEM-3 & $\mathrm{A} / \mathrm{C}$ & $\mathrm{A} / \mathrm{C}$ \\
\hline S36 & K. pneumoniae & $\mathrm{Tc}$ & CTX-M-3/OXA-1 & FII & $\mathrm{F}$ \\
\hline S43 & K. pneumoniae & $\mathrm{Tc}$ & CTX-M-3 & $\mathrm{N}$ & $\mathrm{N}$ \\
\hline S46 & K. pneumoniae & $\mathrm{Tc}$ & CTX-M-15 & $\mathrm{L} / \mathrm{M}$ & $\mathrm{L} / \mathrm{M}$ \\
\hline S51 & K. pneumoniae & $\mathrm{Tc}$ & SHV-12 & FIIK, R & FIIK \\
\hline S59 & K. pneumoniae & $\mathrm{Tc}$ & CTX-M-15 & FIIK & FIIK \\
\hline S75 & K. pneumoniae & $\mathrm{Tc}$ & CTX-M-15/OXA-1 & FIIK & FIIK \\
\hline S82 & K. pneumoniae & $\mathrm{Tc}$ & CTX-M-15/OXA-1 & FII & $\mathrm{F}$ \\
\hline S88 & K. pneumoniae & $\mathrm{Tc}$ & SHV-2a & FIIK & FIIK \\
\hline $\mathrm{S} 90 \dagger$ & K. pneumoniae & Tf & SHV-12, KPC-2 & $\mathrm{X} 3$ & $\mathrm{X} 3$ \\
\hline S00056 & S. enterica Typhimurium & $\mathrm{Tc}$ & CTX-M-2 & HI2 & $\mathrm{HI} \gamma$ \\
\hline S00319 & S. enterica Havana & $\mathrm{Tc}$ & CTX-M-15 & HI2 & $\mathrm{HI} \gamma$ \\
\hline S01106 & S. enterica Virchow & $\mathrm{Tc}$ & SHV-12 & I1 & I1 \\
\hline S01331 & S. enterica Tel el kebir & $\mathrm{Tc}$ & CTX-M-15 & HI2 & $\mathrm{HI} \gamma$ \\
\hline S01477† & S. enterica Typhimurium & $\mathrm{Tc}$ & CTX-M-1/CMY-2 & HI1, I1 & $\mathrm{I} 1, \mathrm{HI} \alpha, \mathrm{HI} \beta$ \\
\hline S01650 & S. enterica Brandeburg & $\mathrm{Tc}$ & CTX-M-14 & FrepB & $\mathrm{F}$ \\
\hline S03207 & S. enterica Typhimurium & Tf & CTX-M-15 & FIA, FIB & $\mathrm{F}$ \\
\hline S03663 & S. enterica Grumpensis & $\mathrm{Tc}$ & CTX-M-15 & HI2 & $\mathrm{HI} \gamma$ \\
\hline S03664 & S. enterica Typhimurium & $\mathrm{Tc}$ & CTX-M-15 & $\mathrm{N}$ & $\mathrm{N}$ \\
\hline S04662 & S. enterica Virchow & $\mathrm{Tc}$ & CTX-M-32 & $\mathrm{N}$ & $\mathrm{N}$ \\
\hline S05343 & S. enterica Concord & $\mathrm{Tc}$ & CTX-M-15 & HI2 & $\mathrm{HI} \gamma$ \\
\hline S07364 & S. enterica Miami & $\mathrm{Tc}$ & SHV-2 & $\mathrm{N}$ & $\mathrm{N}$ \\
\hline S09118 & S. enterica Keurmassar & $\mathrm{Tc}$ & SHV-12 & $\mathrm{HI} 2, \mathrm{FI}$ & $\mathrm{F}, \mathrm{HI} \alpha$ \\
\hline $\mathrm{S} 1922 \dagger$ & S. enterica Kentucky & $\mathrm{Tc}$ & VIM-2 & W & $\mathrm{W}$ \\
\hline S1923 & S. enterica Kentucky & $\mathrm{Tc}$ & VIM-2 & W & $\mathrm{W}$ \\
\hline S27078 & S. enterica Carmel & $\mathrm{Tc}$ & CTX-M-15 & FrepB & $\mathrm{F}$ \\
\hline S7981 & S. enterica Saintpaul & $\mathrm{Tc}$ & OXA-48 & $\mathrm{L} / \mathrm{M}$ & $\mathrm{L} / \mathrm{M}$ \\
\hline
\end{tabular}


Plasmid classification

\begin{tabular}{|c|c|c|c|c|c|c|c|c|c|}
\hline \multirow[b]{2}{*}{ No. } & \multirow[b]{2}{*}{ Species } & \multirow[b]{2}{*}{ B-Lactamase } & \multirow[b]{2}{*}{ PBRT } & \multicolumn{6}{|c|}{ Plasmid relaxase gene typing } \\
\hline & & & & Multiplex I & Multiplex II & Multiplex III & Multiplex IV & Multiplex VI & Simplex I \\
\hline 3 & E. coli & TEM-24 & $\mathrm{A} / \mathrm{C}, \mathrm{B} / \mathrm{O}$ & & $\mathrm{A} / \mathrm{C}$ & & & & $\mathrm{K}-\mathrm{B} / \mathrm{O}$ \\
\hline 15 & E. coli & TEM-24 & $\mathrm{A} / \mathrm{C}, \mathrm{I} 1$ & & $\mathrm{~A} / \mathrm{C}, \mathrm{I} 1$ & & & & \\
\hline 17 & E. coli & TEM-24 & A/C, FIB, FII, HI2, X4 & $\mathrm{F}$ & $\mathrm{A} / \mathrm{C}$ & $\mathrm{HI} \alpha, \mathrm{HI} \gamma$ & $\mathrm{X} 4$ & & \\
\hline 19 & E. coli & TEM-21 & A/C, FIA, FIB, FII, K, N, X1 & & $\mathrm{A} / \mathrm{C}, \mathrm{N}$ & & $\mathrm{X} 1$ & & $\mathrm{~K}-\mathrm{B} / \mathrm{O}$ \\
\hline 23 & E. coli & TEM-24 & A/C, FIB, FII, II & $\mathrm{F}$ & $\mathrm{A} / \mathrm{C}, \mathrm{I} 1$ & & & & \\
\hline 26 & E. coli & TEM-24 & A/C, FIB, HI2 & $\mathrm{F}$ & $\mathrm{A} / \mathrm{C}$ & $\mathrm{HI} \alpha, \mathrm{HI} \gamma$ & & & \\
\hline 28 & E. coli & TEM-21 & $\mathrm{A} / \mathrm{C}, \mathrm{K}, \mathrm{X} 1$ & & $\mathrm{~A} / \mathrm{C}$ & & $\mathrm{X} 1$ & & K-B/O \\
\hline 33 & E. coli & TEM-24 & $\mathrm{A} / \mathrm{C}, \mathrm{P}$ & & $\mathrm{A} / \mathrm{C}$ & & & & \\
\hline 34 & E. coli & TEM-24 & A/C, FIA, FIB, FII, K, N, X1 & & $\mathrm{A} / \mathrm{C}, \mathrm{N}$ & & $\mathrm{X} 1$ & & $\mathrm{~K}-\mathrm{B} / \mathrm{O}$ \\
\hline 40 & E. coli & TEM-24 & $\mathrm{A} / \mathrm{C}, \mathrm{B} / \mathrm{O}, \mathrm{FIA}$ & $\mathrm{F}$ & $\mathrm{A} / \mathrm{C}$ & & & & $\mathrm{K}-\mathrm{B} / \mathrm{O}$ \\
\hline 50 & E. coli & TEM-52 & I1 & & I1 & & & & \\
\hline 53 & E. coli & TEM-3 & A/C, B/O, FrepB & FV & $\mathrm{A} / \mathrm{C}$ & & & & $\mathrm{K}-\mathrm{B} / \mathrm{O}$ \\
\hline 66 & E. coli & SHV-12 & FIB, K & $\mathrm{F}$ & & & & & $\mathrm{K}-\mathrm{B} / \mathrm{O}$ \\
\hline 84 & E. coli & CTX-M-1 & FIB, HI1, N & $\mathrm{F}$ & $\mathrm{N}$ & $\mathrm{HI} \beta$ & & & \\
\hline 88 & E. coli & CTX-M1 & FIB, FII, HII, N & $\mathrm{F}$ & $\mathrm{N}$ & $\mathrm{HI} \beta$ & & & \\
\hline 101 & E. coli & CTX-M-2 & $\mathrm{HI} 2, \mathrm{P}$ & & & $\mathrm{HI} \alpha, \mathrm{HI} \gamma$ & & & \\
\hline 105 & E. coli & CTX-M-1 & $\mathrm{K}, \mathrm{L} / \mathrm{M}$ & & L/M, I1 & & & & $\mathrm{K}-\mathrm{B} / \mathrm{O}$ \\
\hline 106 & E. coli & CTX-M-1 & FIB, FII, HII, N & $\mathrm{F}$ & $\mathrm{N}$ & $\mathrm{HI} \alpha, \mathrm{HI} \beta$ & & & \\
\hline 112 & E. coli & CTX-M-3 & FIB, FII, K & $\mathrm{F}$ & & & & & K-B/O \\
\hline Ec37040 & E. coli & ND & A/C, FIB, FII & $\mathrm{F}$ & $\mathrm{A} / \mathrm{C}$ & & & & \\
\hline Ec43681 & E. coli & ND & FIA, FIB, FII, Q & $\mathrm{F}$ & & & & Q1 & \\
\hline KpS3 & K. pneumoniae & FOX-3 & $\mathrm{A} / \mathrm{C}, \mathrm{FIIK}, \mathrm{X} 4$ & FIIK & $\mathrm{A} / \mathrm{C}$ & & $\mathrm{X} 4$ & & \\
\hline KpS5 & K. pneumoniae & DHA-1 & FIIK, L/M, R & FIIK & $\mathrm{L} / \mathrm{M}$ & & & & \\
\hline KpS15 & K. pneumoniae & GES-9 & FIIK, R, U & FIIK & & & & & \\
\hline $\mathrm{KpS} 19$ & K. pneumoniae & CTX-M-15, DHA-1 & FII, FIIK, L/M, N & FIIK, $\mathrm{F}$ & $\mathrm{L} / \mathrm{M}, \mathrm{N}$ & & & & \\
\hline KpS20 & K. pneumoniae & CTX-M-15,SHV-2a & FII, X4 & $\mathrm{F}$ & LAVI, IV & & $\mathrm{X} 4$ & & \\
\hline KpS26 & K. pneumoniae & SHV-12 & FIIK, N, R & FIIK & $\mathrm{N}$ & & & & \\
\hline KpS47 & K. pneumoniae & CTX-M-3 & $\mathrm{HI} 2, \mathrm{~L} / \mathrm{M}$ & & $\mathrm{L} / \mathrm{M}$ & $\mathrm{HI} \gamma$ & & & \\
\hline $\mathrm{KpS63}$ & K. pneumoniae & DHA-1 & $\mathrm{L} / \mathrm{M}, \mathrm{R}$ & FIIK & $\mathrm{L} / \mathrm{M}$ & & & & \\
\hline KpS83 & K. pneumoniae & TEM-3 & $\mathrm{A} / \mathrm{C}, \mathrm{FIB}, \mathrm{N}$ & $\mathrm{F}$ & $\mathrm{A} / \mathrm{C}, \mathrm{N}$ & & & & \\
\hline KpS88 & K. pneumoniae & SHV-2a & FIIK & FIIK & & & & & \\
\hline KpS91 & K. pneumoniae & KPC-2 & A/C, FIIK, X3 & FIIK & $\mathrm{A} / \mathrm{C}$ & & $\mathrm{X} 3$ & & \\
\hline KpS92 & K. pneumoniae & CTX-M-14, VIM-1 & FII, FIIK, II & FIIK, $\mathrm{F}$ & Il & & & & \\
\hline KpS93 & K. pneumoniae & CTX-M-15, OXA-48 & $\mathrm{I} 1, \mathrm{~L} / \mathrm{M}$ & & $\mathrm{I} 1, \mathrm{~L} / \mathrm{M}$ & & & & \\
\hline FM-2 & K. pneumoniae & OXA- 48 & FIIK, L/M, N & FIIK & $\mathrm{L} / \mathrm{M}, \mathrm{N}$ & & & & \\
\hline FM-10 & K. pneumoniae & OXA-48 & $\mathrm{A} / \mathrm{C}, \mathrm{L} / \mathrm{M}$ & & $\mathrm{A} / \mathrm{C}, \mathrm{L} / \mathrm{M}$ & $\mathrm{HI} \alpha$ & & & \\
\hline Кр29537 & K. pneumoniae & ND & A/C, FIIK & FIIK & $\mathrm{A} / \mathrm{C}$ & & & & \\
\hline S10-1477 & S. enterica Typhimurium & CTX-M-1, CMY-2 & HI1, I1 & & I1 & $\mathrm{HI} \alpha, \mathrm{HI} \beta$ & & & \\
\hline S10-1526 & S. enterica Typhimurium & CTX-M-1, CMY-2 & HI1, I1 & & I1 & $\mathrm{HI} \alpha, \mathrm{HI} \beta$ & & & \\
\hline
\end{tabular}


Table 4 - Results of relaxase gene typing in 17 recipient cells non-typeable with PBRT $^{\star}$

\begin{tabular}{|c|c|c|c|c|}
\hline No. & Parental species & $\mathrm{Tc} / \mathrm{Tf}$ & $\beta$-lactamase & $\begin{array}{l}\text { Plasmid relaxase gene } \\
\text { typing }\end{array}$ \\
\hline 16 & E. coli & $\mathrm{Tc}$ & TEM-21 & $\mathrm{C} 11$ \\
\hline 31 & E. coli & $\mathrm{Tc}$ & TEM-24 & P5-3 \\
\hline 37 & E. coli & $\mathrm{Tf}$ & TEM-24 & P5-3 \\
\hline 55 & E. coli & $\mathrm{Tf}$ & TEM-52 & NT \\
\hline 65 & E. coli & $\mathrm{Tc}$ & SHV-4 & P5-3 \\
\hline 66 & E. coli & $\mathrm{Tc}$ & SHV-12 & P5-3 \\
\hline 67 & E. coli & $\mathrm{Tc}$ & SHV-12 & P5-3 \\
\hline 70 & E. coli & $\mathrm{Tc}$ & SHV-12 & P5-3 \\
\hline 71 & E. coli & $\mathrm{Tc}$ & SHV-2 & P5-3 \\
\hline 72 & E. coli & $\mathrm{Tc}$ & SHV-2 & P5-3 \\
\hline 76 & E. coli & $\mathrm{Tc}$ & SHV-2 & $\mathrm{NT}, \mathrm{P} 5-3 \uparrow$ \\
\hline 86 & E. coli & $\mathrm{Tf}$ & CTX-M-3 & NT \\
\hline 93 & E. coli & $\mathrm{Tf}$ & CTX-M-3 & NT \\
\hline 99 & E. coli & $\mathrm{Tc}$ & CTX-M-1 & $\mathrm{Qu}, \mathrm{P} 5-3 \dagger$ \\
\hline S33 & K. pneumoniae & $\mathrm{Tf}$ & DHA-1 & NT \\
\hline S55 & K. pneumoniae & $\mathrm{Tc}$ & CTX-M-15, OXA-1 & FIIK \\
\hline S77 & K. pneumoniae & $\mathrm{Tf}$ & SHV-12 & NT \\
\hline
\end{tabular}

390

*PBRT, PCR based replicon typing, Tc, transconjugant, Tf, transformant, NT, not typeable; $\uparrow$ : recipient cells containing two different plasmids (ESBL was carried by plasmids NT and Qu in strains 76 and 99 respectively). 
Supplementary Table S1 - Relaxase and T4SS-ATPase gene sequences of completely sequenced IncHI plasmids stored in GenBank

\section{Supplementary Table S2 - Primers used in this study for T4SS-typing}

401

402

403

404

405

406

407

408

409

410

411

412

413

414

415

416

417

418

419

420

421

422

423

424

\section{Supplementary Figure - Relaxase gene typing assays}

All PCR products were separated in $1.5 \%$ agarose gels. M, molecular size marker (in bp). (a) Multiplex PCR assay of the IncF, IncFIIK and IncFV relaxase genes. Lanes : $1, K$. pneumoniae $\mathrm{KpS19}$; 2, E. coli 84 ; 3, E. coli 53 ; 4, K. pneumoniae KpS83 ; 5, K. pneumoniae $\mathrm{KpS} 19+$ E. coli 53 ; 6, E. coli J53 (negative control). (b) Multiplex PCR assay of the IncA/C, IncI1, IncL/M and IncN relaxase genes. Lanes : 1, E. coli $15 ; 2$, E. coli $34 ; 3, K$. pneumoniae $\mathrm{KpS} 19$; 4, K. pneumoniae $\mathrm{FM} 10$; 5, E. coli $15+$ E. coli 34+ K. pneumoniae KpS19; 6, E. coli J53 (negative control). (c) Multiplex PCR assay of the Hia, Hi $\beta$ and HI $\gamma$ relaxase genes. Lanes : 1, K. pneumoniae FM10 ; 2, E. coli 26 ; 3, E. coli 88 ; 4, E. coli 106 ; 5, E. Coli 17+E. Coli $106 ; 6$, E. coli J53 (negative control). (d) Multiplex PCR assay of the IncX1, IncX2, IncX3 and IncX4 relaxase genes. Lanes: 1, E. coli 17; 2, K. pneumoniae S90 recipient cell; 3, E. coli ECOR19; 4, E. coli 19; 5, E. coli J53 (negative control). (e) Multiplex PCR assay of the MobP5-1, MobP5-2, MobP5-3, MobC11 and MobQu relaxase genes. Lanes : 1, E. coli ECOR6 ; 2, E. coli ECOR10 ; 3, E. Coli 16 recipient cell ; 4, E. Coli 99 recipient cell ; 5, E. coli ECOR6+ E. coli ECOR10+E. Coli 16 recipient cell+E. Coli 70 recipient cell+ E. Coli 99 recipient cell ; 6, E. coli J53 (negative control). (f) Multiplex PCR assay of the IncP1 $\alpha$, IncQ1, IncU and IncW relaxase genes. Lanes : 1, E. coli Ec43681; 2, S. enterica S1922 recipient cell ; 3, pFBAOT6 reference plasmid ; 4, RP4 reference plasmid ; 5, E. coli Ec43681+ pFBAOT6 reference plasmid+ RP4 reference plasmid ; 6, E. coli J53 (negative control). (g) Simplex PCR assay of the IncB/O and IncK relaxase genes. Lanes : 1, E. coli 34 ; 2, E. coli 66 ; 3, E. coli $40 ; 4$, E. Coli 112 ; 5, E. coli 53 ; 6, E. coli J53 (negative control). 